\title{
Rate Region Analysis of Multi-terminal Neuronal Nanoscale Molecular Communication Channel
}

\author{
Hamideh Ramezani, Student Member, IEEE, Caglar Koca, Student Member, IEEE and \\ Ozgur B. Akan, Fellow, IEEE
}

\begin{abstract}
In this paper, we investigate the communication channel capacity among hippocampal pyramidal neurons. To this aim, we study the processes included in this communication and model them with realistic communication system components based on the existing reports in the physiology literature. We consider the communication between two neurons and reveal the effects of the existence of multiple terminals between these neurons on the achievable rate per spike. To this objective, we derive the power spectral density (PSD) of the signal in the output neuron and utilize it to calculate the rate region of the channel. Moreover, we evaluate the impacts of vesicle availability on the achievable rate by deriving the expected number of available vesicles in input neuron using a realistic vesicle release model. Simulation results show that number of available vesicles for release does not affect the achievable rate of neuro-spike communication with univesicular release model. However, in neurons that multiple vesicles can release from each synaptic terminal, achievable rate is significantly affected by depletion of vesicles. Moreover, we show that increasing the number of synaptic terminals between two neurons makes the synaptic connection stronger. Hence, it is an important factor in learning and memory, which occur in the hippocampal region of the brain based on the synaptic connectivity.
\end{abstract}

\section{INTRODUCTION}

Nanomachines have limited capabilities in computing, data storing, sensing and actuation as a result of their size. Hence, they need to establish networks with each other to become capable for more complex tasks. Among the proposed paradigms for nanonetworks, molecular communication, in which molecules are used to encode, transmit and receive information, is the most promising paradigm since this communication exists in the structure of any living organism and is a biocompatible and biostable solution [1]. One of the significant mechanisms for molecular communication inside the human body is the ultra-large scale network of nerve cells, i.e., neurons, which is known as nanoscale neuro-spike communication [2]. Realistically modeling, analyzing and understanding communication theoretical capabilities of the neuro-spike communication channel contribute to the development of bio-inspired solutions for nanonetworks and ICTinspired solutions for neural diseases caused by dysfunction

H. Ramezani and C. Koca are with the Next-generation and Wireless Communications Laboratory (NWCL), Department of Electrical and Electronics Engineering, Koc University, Istanbul, Turkey, (e-mails: hramezani13@ku.edu.tr and cagkoca@ku.edu.tr).

O. B. Akan is with Internet of Everything (IoE) Group, Electrical Engineering Division, Department of Engineering, University of Cambridge, UK, (e-mail: oba21@cam.ac.uk).

This work was supported in part by ERC project MINERVA (ERC-2013CoG \#616922), EU project CIRCLE (EU-H2020-FET-Open \#665564), and TŬBITTAK graduate scholarship program (BIDEB-2215).

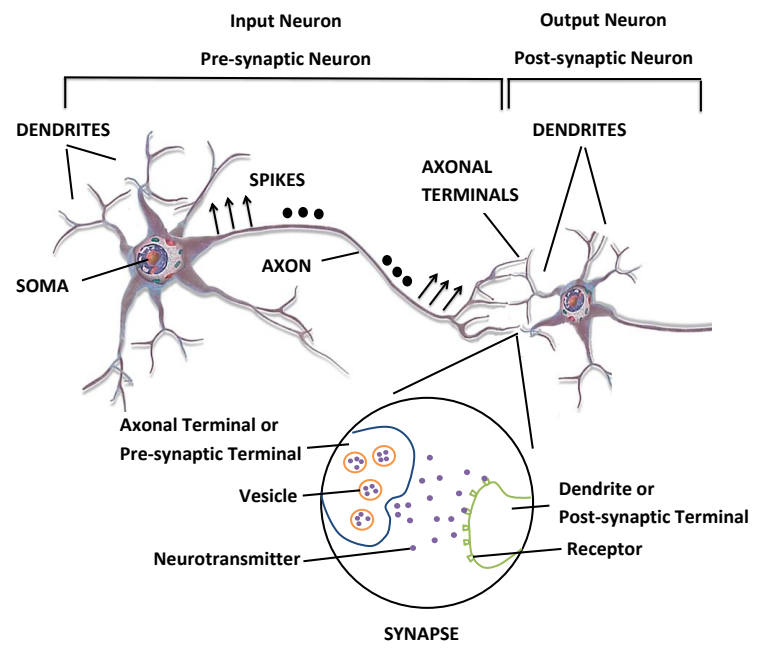

Fig. 1. Neural anatomy of communication among two neurons.

of intra-body nanonetworks [3].

The fundamental processes of neuro-spike communication are depicted in Fig. 1 [4]. Main parts of each neuron participating in this communication are (i) dendrites, receivers of a neuron, where the signal is received as positive or negative changes in the membrane potential, (ii) soma, the processing unit where the decision of receiving a signal is made, (iii) axon, the communication channel between soma and pre-synaptic terminals, and (iv) pre-synaptic terminals, the transmitting points of the neuron. The input neuron encodes its information in spike trains, which are transmitted to the pre-synaptic terminals through axon. Arrival of a spike to the pre-synaptic terminal initiates release of vesicles containing a group of neurotransmitters to the synapse, i.e., the gap between input and output neuron. These neurotransmitters then diffuse through synapse until reaching output neuron, where they bind to the receptors of the output neuron and cause changes in its membrane potential.

There has been some research concentrating on functionality of neurons from the perspective of communication theory. One such a study is [2], where an end-to-end communication channel model is introduced for neuro-spike communication. Another model is given in [5], where the biological processes within this communication are modeled by their frequency response. The fundamental events during synaptic transmission are considered in [6], where the capacity lower bounds of a point-to-point synaptic transmission is derived by signal estimation and detection paradigms. The performance of 


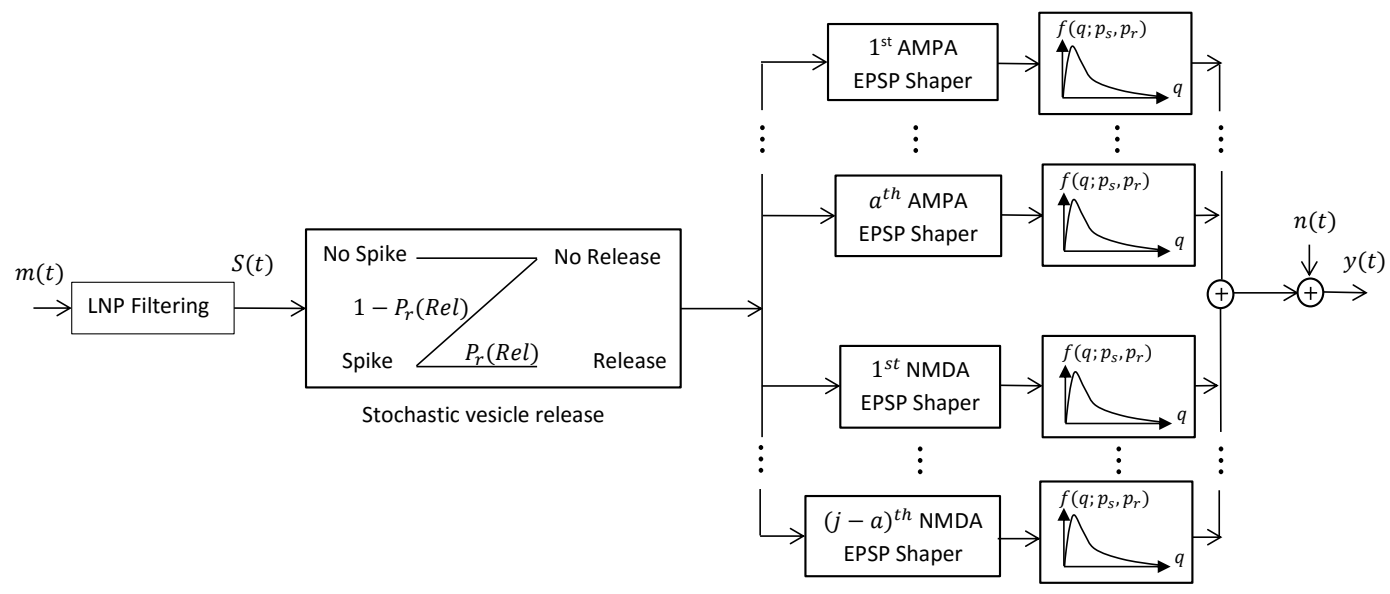

Fig. 2. Neuro-spike communication model for information transmission among two hippocampal pyramidal neurons.

spike detection in neuro-spike communication with axonal noise is studied in [7]. In [8], [9], the achievable rate of this communication is studied under different channel models. The multiple-access among hippocampal-cortical neurons is considered in [8], where the effect of the number of presynaptic neurons is studied on the achievable post-synaptic rate. The ergodic capacity of the Multiple-Input MultipleOutput (MIMO) synaptic communication channel is derived in [10]. Although all of the these studies consider existence of one synaptic connection between an input and output neuron, multiple synapses may exist between a pair of hippocampal pyramidal neurons as it is shown in [11], [12]. Hence, these models cannot accurately capture the communication capabilities of hippocampal pyramidal neurons.

The main contribution of our work is deriving information rate of communication among hippocampal pyramidal neurons using a more realistic channel model. For this objective, we utilize a realistic pool-based synapse model and consider the existence of multiple synapses between an input and output neuron. Our motivation is that the learning and memory, which occur in the hippocampal region of the brain, are based on the synaptic connectivity and its strength. Hence, in this paper, we perform analysis to observe how the vesicle release process, vesicle pool features, and existence of multiple terminals between two neurons affect the strength of the overall synaptic connection. Results of this study give us insight on design of communication paradigms for nanomachines, implementation of learning and memory in artificial neurons and realization of artificial neural systems.

The remainder of this paper is organized as follows. In Section II, a realistic channel model for neuro-spike communication is explained. Then, the power spectral density (PSD) of the signal and noise components in the post-synaptic neuron are calculated in Section III. Moreover, derived PSDs are used to obtain the rate region of communication among these neurons. In Section IV then, the total achievable rate per spike at the post-synaptic neuron of the introduced communication channel model is quantitatively compared with the existing channel models with single synaptic terminal to find the effect of increasing the number of terminals between a pair of neurons on the achievable rate. Moreover, the dependence of the achievable rate on dynamics of vesicle pool size and vesicle release process are investigated through simulations. Finally, the paper is concluded in Section V.

\section{Neuro-SPike CommuniCATION Model}

As shown in Fig. 2, we utilize a mathematical model for point to point neuro-spike communication among a pair of hippocampal pyramidal neurons. The input of this communication channel is the environmental stimulus shown by $m(t)$ in Fig. 2, which is modeled by a wide-sense stationary (WSS) process with bandwidth $B_{m}$ in [8]. The neural response to this stimulus is modeled by a linear-nonlinear-Poisson (LNP) filter in [13], where $m(t)$ is first passed from a linear weighting filter, whose output is derived in [8] by

$$
v(t)=m(t) * k(t)=m(t) *\left[\frac{1}{C} \exp \left(-\frac{t}{R C}\right) u(t)\right] .
$$

In (1), $u(t)$ and $k(t)$ are the Heaviside step function and a low-pass filter with parameters $R$ and $C$ corresponding to the soma resistance and capacitance, respectively. Then, $v(t)$ is passed through a point non-linearity block given by

$$
f(v(t))=\frac{1}{1+\exp \left[-a_{0}\left(v(t)-v_{\frac{1}{2}}\right)\right]},
$$

where $a_{0}$ and $v_{\frac{1}{2}}$ are firing rate control parameter and voltage at half the maximum firing rate, respectively. Finally, the response of the neuron to stimulus, i.e., spike train, is generated by a Poisson distribution with inhomogeneous spike rate defined as $\lambda(t)=\lambda_{\max } f(v(t))$. The Poisson spike train, $S(t)$, can be formulated as $S(t)=\sum_{h=1}^{N_{\text {Pois }}(t)} \delta\left(t-t_{h}\right)$, where $t_{h}$ is the occurrence time of the $h^{t h}$ spike and the mean of the Poisson arrival depends on the input, $\lambda(t)$, by $E\left[N_{\text {Pois }}(t)\right]=\int_{0}^{t} \lambda(\tau) d \tau$.

The spike train, i.e., $S(t)$, is then transmitted to presynaptic terminals through axon. Although the shape of the 


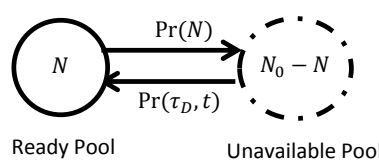

Fig. 3. Pool-based model for vesicle release from a synaptic terminal.

spike may change during axonal transmission [14], more experimental data is needed to accurately model functionality of axon [15]. Hence, we consider the axon as an ideal filer, which is a fairly accurate model for hippocampal pyramidal neurons since their axonal transmission is highly reliable due to the existence of myelin sheaths and Node of Ranviers [5].

Arrival of each spike to the pre-synaptic terminal initiates release of vesicles to the synapse. Since after each vesicle release only the reserve vesicles that are closest to the release site have a significant probability for filling the vacancy [16], we use the single-pool model shown in Fig. 3 for vesicle release, where the ready pool (RP) has a limited capacity $N_{0}$, which is the number of readily releasable vesicles (RRVs) in this pool when the neuron is not stimulated for a long time and all RRVs are refilled, and each vacancy is refilled at a rate independent of the reserve pool size. Moreover, $\operatorname{Pr}(N)$ is the release probability per stimulus when the RP has $N$ vesicles with $N \leq N_{0}, \tau_{D}$ is the mean recovery time of a vacancy replenishment, and $\operatorname{Pr}\left(\tau_{D}, t\right)$ is the probability of one vacancy replenishment after $t$ second. Since the number of vesicles ready to be docked in neurons is much higher than $N_{0}$ [16], the recovery of a vacancy can be modeled by the first event from a Poisson process [17]. Hence, vacancy replenishment probability can be written as $\operatorname{Pr}\left(\tau_{D}, t\right)=1-\exp \left(-\frac{t}{\tau_{D}}\right)$ and the expected number of vacancy replenishments is $\left(N_{0}-N\right)\left(1-\exp \left(-\frac{t}{\tau_{D}}\right)\right)$.

Based on the biological studies, only one vesicle can be released per action potential from each release terminal in hippocampal pyramidal neurons [18]. However, multiple synapses may exist between a pair of hippocampal pyramidal neurons [11], [12], which results in the release of more than one vesicle to all synapses between two neurons. Hence, we consider the existence of multiple synaptic terminals between an input and output neuron and use univesicular release constraint for each terminal. As shown in the stochastic vesicle release block of Fig. 2, the release of vesicles after arrival of each spike to the pre-synaptic terminal occurs with probability $P_{r}(R e l)$, which is derived by

$$
\operatorname{Pr}(\operatorname{Rel})=\sum_{k=1}^{n_{t}} \operatorname{Pr}(k \text { release }),
$$

where $\operatorname{Pr}(k$ release $)$ is the probability of releasing $k$ vesicles from $n_{t}$ terminals. As we have shown in [19], $\operatorname{Pr}(k$ release $)$ can be derived from Poisson Binomial distribution with mean, $\mu=\sum_{i=1}^{n_{t}} P_{r}\left(N_{i}\right)$, and variance, $\sigma^{2}=\sum_{i=1}^{n_{t}} P_{r}\left(N_{i}\right)(1-$ $\left.\operatorname{Pr}_{r}\left(N_{i}\right)\right)$, where $\operatorname{Pr}\left(N_{i}\right)=1-\exp \left(-N_{i} \alpha_{v}\right)$ is the probability of release of one vesicle from $i$ th pre-synaptic terminal when $N_{i}$ vesicles are ready to be released in the terminal and the release rate can be calculated by $\alpha_{v}=\int \lambda(t) d t$, where the integral is taken over duration of the spike [8].

For the next spike arrival, we update the number of RRVs. The expected number of releases from a terminal with $N_{i}$ RRVs is equal to $\operatorname{Pr}\left(N_{i}\right)$. Hence, after arrival of a spike, the expected number of RRVs becomes $N_{i}-\operatorname{Pr}\left(N_{i}\right)$. If we consider $\tau_{D, i}$ and $N_{0, i}$ as the mean recovery time of the vacancy replenishment and the capacity of RP in $i$ th synaptic terminal, the expected number of RRVs for next spike arrival in this synaptic terminal becomes

$$
\begin{aligned}
N_{i}^{n} & =N_{i}-\operatorname{Pr}\left(N_{i}\right) \\
& +\left(N_{0, i}-N_{i}+\operatorname{Pr}\left(N_{i}\right)\right)\left(1-\exp \left(-\frac{\Delta t}{\tau_{D, i}}\right)\right),
\end{aligned}
$$

where $\Delta t$ is the time interval between two spikes.

Released neurotransmitters diffuse through synapses towards the receptors of the post-synaptic neuron. Their binding to these receptors stimulates the post-synaptic neuron. Since the response of AMPA and NMDA receptors are dominant [4], and the ratio of the ionic flow entering the post-synaptic neuron through AMPA and NMDA receptors is constant for different synapses to a neuron [20], we neglect other receptors in our model and choose the average number of bound AMPA receptors equal to a fixed ratio, $0 \leq r \leq 1$, of total bound receptors. The response of AMPA and NMDA receptors is modeled with an alpha function in [2] as

$$
\alpha(t, \tau)=h_{\max } \frac{t}{\tau} \exp \left(1-\frac{t}{\tau}\right) u(t),
$$

where $\tau$ is a constant related to type of the receptor and $h_{\max }$ is the maximum of the response. We define $\alpha_{A}(t)=\alpha\left(t, \tau_{1}\right)$, and $\alpha_{N}(t)=\alpha\left(t, \tau_{2}\right)$, for AMPA, and NMDA receptors, respectively, with $\tau_{2}>\tau_{1}$.

To model the trial-to-trial variability in the maximum amount of the membrane potential change in the postsynaptic neuron upon release of a vesicle, we multiply the amplitude of the response of each receptor with a random variable $q$ with probability distribution of $P(q)$ [6]. $P(q)$ is modeled by a gamma distribution in the literature [8] as

$$
f\left(q ; p_{s}, p_{r}\right)=\frac{p_{r}}{\Gamma\left(p_{s}\right)}\left(p_{r} q\right)^{p_{s}-1} \exp \left(-p_{r} q\right)
$$

with $q \geq 0$ and $p_{s}, p_{r}>0$, where $p_{s}$ and $p_{r}$ are shape and rate parameters of gamma distribution, respectively, and $\Gamma\left(p_{s}\right)$ is the gamma function and is calculated by

$$
\Gamma\left(p_{s}\right)=\int_{0}^{\infty} x^{p_{s}-1} e^{-x} d x .
$$

As we model trial-to-trial variability with random variable $q$, we assume that a fixed number of released neurotransmitters from each vesicle, $N_{N t}$, bind to the receptors of output neuron. Hence, release of $K$ vesicles to all synapses among two neurons causes receiving of $j=N_{N t} K$ neurotransmitters with $a=\lfloor r j\rfloor$ of them bound to AMPA receptors, where $\lfloor r j\rfloor$ is the largest integer number less than $r j$.

Post-synaptic membrane voltage is corrupted with noise sources like thermal noise, channel noise and synaptic noise due to the multiple access to the synapse from thousands 
of neurons. Since the signal of different neurons has the same random structure, probability density function of the post-synaptic noise, shown by $n(t)$ in Fig. 2, converges to a Gaussian process due to the central limit theorem [2], [6].

\section{Achievable Rate Region Analysis}

In this work, we evaluate the performance of the neurospike communication based on the achievable rate upon arrival of a spike to the pre-synaptic terminals at time $t_{h}$. The received signal in the post-synaptic neuron can be written as

$$
y(t)=\sum_{i=1}^{n_{t}}\left(\sum_{k=1}^{N_{N t}}\left(q_{i k} \alpha_{i k}\left(t-t_{h}\right)\right) x_{i}\right)+n(t),
$$

where $q_{i k}$ and $n(t)$ are variable quantal amplitude for $k$ th neurotransmitter released from $i$ th terminal and noise in the post-synaptic neuron, respectively, and

$$
\begin{gathered}
\alpha_{i k}(t)= \begin{cases}\alpha_{A}(t), & \text { AMPA receptors } \\
\alpha_{N}(t), & \text { NMDA receptors }\end{cases} \\
x_{i}= \begin{cases}0, & 1-\operatorname{Pr}\left(N_{i}\right) \\
1, & \operatorname{Pr}\left(N_{i}\right)\end{cases}
\end{gathered}
$$

Our objective is to find the achievable rate per spike in the post-synaptic terminal. Hence, we calculate the PSD of the signal and noise received at the post-synaptic neuron.

In [8], with simplifying point non-linearity of LNP filter, PSD of the time averaged version of $S(t)$, which is a WSS process if we assume that $S(t)$ is ergodic, is calculated as

$$
S_{\bar{S}}(f)=\bar{\lambda}^{2} \delta(f)+(\bar{\lambda} b)^{2} S_{v}(f)+\bar{\lambda},
$$

where PSD of $v(t)$, i.e., $S_{v}(f), b$ and the mean firing rate, i.e., $\bar{\lambda}$ are defined as follows.

$$
\begin{gathered}
S_{v}(f)=\frac{R^{2}}{1+(2 \pi f R C)^{2}} S_{m}(f) \\
b=\frac{a_{0} \lambda_{\max }}{4 \lambda} P\left(\left|v(t)-v_{\frac{1}{2}}\right| \leq \frac{2}{a_{0}}\right) \\
\bar{\lambda}=\lambda_{\max }\left(\frac{2-a_{0} v_{\frac{1}{2}}}{4}\right) P\left(\left|v(t)-v_{\frac{1}{2}}\right| \leq \frac{2}{a_{0}}\right) \\
+\lambda_{\max } P\left(v(t)-v_{\frac{1}{2}} \geq \frac{2}{a_{0}}\right)
\end{gathered}
$$

In (12)-(14), $S_{m}(f)$ is the PSD of $m(t)$, which has a zero mean normal distribution with variance $\sigma_{m}^{2}$, and $P(A)$ depicts the probability of occurrence of event $A$.

Same as [8], we assume that the reserve pool refills released RRVs immediately and derive the achievable rate of the communication. Then, for the first time in the literature, we evaluate impacts of ready pool statistics on achievable rate in next section, where we conclude that this assumption does not affect the achievable rate of channel with multiple synaptic terminals when univesicular release model is used. Hence, the closed form equation that we derive in this section gives the achievable rate of this communication even when vesicle pool statistics are realistically modeled.
The arrival of action potentials to the pre-synaptic terminals has Poisson distribution with rate $\lambda(t)$ and the expected number of released vesicles per action potential is $P_{r}\left(N_{0, i}\right)$ for $i$ th synaptic connection. Hence, the vesicles released from $i$ th pre-synaptic terminal has Poisson distribution with rate $P_{r}\left(N_{0, i}\right) \lambda(t)$. Thus, PSD of vesicle release from $i$ th terminal, for $1 \leq i \leq n_{t}$, can be obtained from (11) as

$S_{V R}^{i}(f)=\left(P_{r}\left(N_{0, i}\right)\right)^{2}\left[\bar{\lambda}^{2} \delta(f)+(\bar{\lambda} b)^{2} S_{v}(f)\right]+P_{r}\left(N_{0, i}\right) \bar{\lambda}$.

Moreover, PSD of $w_{i k}(t)$, i.e., the signal of $k$ th released neurotransmitter from $i$ th terminal after scaling with variable quantal amplitude $q_{i k}$, can be written as

$$
\begin{aligned}
S_{w_{i k}}(f)= & \left(\bar{q} P_{r}\left(N_{i}\right)\right)^{2}\left[\bar{\lambda}^{2} \delta(f)+(\bar{\lambda} b)^{2} S_{v}(f)\right] \\
& +\left(\sigma^{2}+\bar{q}^{2}\right) P_{r}\left(N_{i}\right) \bar{\lambda} \\
& =S_{w_{i}}(f),
\end{aligned}
$$

where $1 \leq k \leq N_{N t}$. Hence, the PSD of the output is derived as

$$
S_{y}(f)=\sum_{i=1}^{n_{t}} S_{w_{i}}(f) \sum_{k=1}^{N_{N t}}\left|H_{i k}(f)\right|^{2}+S_{n_{0}}(f),
$$

where $H_{i k}(f)$ is the Fourier transform of $\alpha_{i k}(t)$ and $S_{n_{0}}(f)$ is the PSD of the noise $n(t)$. Hence, the channel rate region can be achieved by

$$
\text { rate }<\int_{-B / 2}^{B / 2} \log \left(1+\frac{\sum_{i=1}^{n_{t}} S_{w_{i}}(f) \sum_{k=1}^{N_{N t}}\left|H_{i k}(f)\right|^{2}}{S_{n_{0}}(f)}\right) d f .
$$

\section{Performance Evaluation}

To find the effect of our vesicle release model on the performance of the neuro-spike communication, we compare the achievable rate of our communication model with models considering existence of single terminal with univesicular and multivesicular release [8], [9] by using the numerical parameters given in Table I. Two factors are important in the achievable rate: (i) average release probability of a vesicle, i.e., $P_{v}=1-\exp \left(-\alpha_{v}\right)$, and (ii) number of RRVs. To investigate the dependence of the achievable rate of this communication on $P_{v}$, we utilize (17), where variations in the number of RRVs as a result of vesicle release are ignored. Then, we analyze variations in the number of RRVs by utilizing the updating mechanism of expected number of RRVs given in (4) and reveal the impacts of these variations on the achievable rate by simulations.

\section{A. Dependency of Achievable Rate on Release Probability}

The achievable rate for different vesicle release probabilities by utilizing a fixed number of RRVs for different terminals is illustrated in Fig. 4. The outcomes of this figure can be itemized as follows.

- Increasing the probability of vesicle release, $P_{v}$, improves the achievable rate of all models. 
TABLE I

SIMULATION PARAMETERS FOR SYNAPTIC CHANNEL MODEL

\begin{tabular}{|c|c|c|c|}
\hline Parameter & Symbol & Value & Ref. \\
\hline Mean and variance of white Gaussian stimulation & $E[m]$ and $\sigma_{m}^{2}$ & 0 and 1 & {$[8]$} \\
\hline Number of synaptic terminals & $n_{t}$ & Between 1 and 5 & {$[12]$} \\
\hline Firing rate control parameter and maximum firing rate & $a_{0}$ and $\lambda_{\max }$ & $\frac{1}{0.029}$ and $36.03 \mathrm{~Hz}$ & {$[8]$} \\
\hline Voltage at half the maximum firing rate & $v_{\frac{1}{2}}$ & $0.036 \mathrm{~V}$ & [8] \\
\hline Mean and variance of quantal amplitude & $E[q]$ and $\operatorname{Var}[q]$ & $\frac{1}{N_{N t}}$ and $\left(\frac{0.6}{N_{N t}}\right)^{2}$ & {$[8]$} \\
\hline $\begin{array}{l}\text { Mean, Variance and maximum of the capacity of } R P \text { in } \\
i^{\text {th }} \text { terminal }\end{array}$ & $E\left[N_{0, i}\right], \operatorname{Var}\left[N_{0, i}\right], \max \left[N_{0, i}\right]$ & $10.3,5.6^{2}$, and 27 & {$[21]$} \\
\hline Mean recovery time constant of $i^{\text {th }}$ terminal & $\tau_{D, i}$ & $\frac{0.6}{N_{0, i}} \mathrm{~s}$ & {$[17]$} \\
\hline Number of bound neurotransmitters per vesicle & $N_{N t}$ & 11.3 & [22] \\
\hline Fraction of AMPA receptors & $r$ & 0.72 & {$[22]$} \\
\hline Peak magnitude and time to peak for the EPSP of AMPAs & $h_{\max , A}$ and $\tau_{1}$ & $1 \mathrm{mV}$ and $8 \mathrm{~ms}$ & {$[2]$} \\
\hline Peak magnitude and time to peak for the EPSP of NMDAs & $h_{\max , N}$ and $\tau_{2}$ & $1 \mathrm{mV}$ and $10 \mathrm{~ms}$ & {$[2]$} \\
\hline Mean, variance, and bandwidth of $A W G N$ & $E[n], \operatorname{Var}[n]$, and $B_{n}$ & $0,0.01 \mathrm{~V}, 100 \mathrm{~Hz}$ & {$[8]$} \\
\hline
\end{tabular}

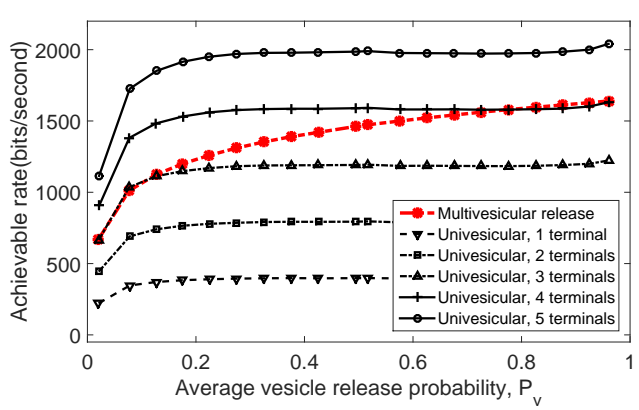

Fig. 4. Effect of average vesicle release probability on the achievable rate while ignoring changes in the number of RRVs.

- In models with univesicular release, only one vesicle can be released from each terminal per spike. Hence, the achievable rate saturates by increasing the probability of vesicle release, $P_{v}$. However, in the synaptic terminal with multivesicular release, all vesicles are free to release upon arrival of one spike. Hence, the expected number of vesicle releases, thus, the achievable rate of the neuro-spike communication, increases by vesicle release probability, $P_{v}$. Thus, the increase in the achievable rate due to increase in vesicle release probability, $P_{v}$, is more in synaptic terminal with multivesicular release model.

- The number of vesicles released, thus, the achievable rate, increase with the number of terminals. Hence, existence of multiple terminals between two neurons strengthens the synaptic connectivity.

\section{B. Dependency of Achievable Rate on Availability of Vesicles}

Next step is finding how the variation in the number of RRVs due to the vesicle depletion affects the achievable rate. To reach this aim, we reveal the variations in the number of RRVs for a synaptic terminal under univesicular and multivesicular release scenarios first. Then, we find the achievable rate by using simulation in which the number of RRVs vary by each vesicle release and vacancy replenishment.

To find the effects of univesicular and multivesicular release scenarios on the number of RRVs, we consider that a synaptic terminal has $10 \mathrm{RRVs}$, which is the nearest integer to the mean of the capacity of RP stated in Table I, then we assume that the probability of vesicle release per spike, $P_{v}$, is constant in the terminal and give spike train with different $\lambda$, selected based on the simulation results reported in [8], as input of this synaptic terminal. The expected number of RRVs for univesicular and multivesicular release models with different amount of release probability per spike, $P_{v}$, are shown in Fig. 5. Based on this figure, (i) the expected number of RRVs decreases by increasing the probability of vesicle release per spike, $P_{v}$, (ii) the number of RRVs in a synaptic terminal under univesicular release model is higher than multivesicular release model since the expected number of releases per spike from each terminal is higher in multivesicular release model and the neuron does not have enough time to refill the pool of RRVs, and (iii) increasing rate of spike train, i.e., $\lambda$, decreases the number of RRVs in both univesicular and multivesicular release scenarios since the neuron has lower recovery time at higher rates, moreover, it has a stronger affect on the multivesicular release model.

Finally, the achievable rate of neuro-spike communication for different values of the vesicle release probability, $P_{v}$, when number of RRVs vary due to the vesicle release and vacancy replenishment is shown in Fig. 6. By comparing Fig. 4 and Fig. 6, we conclude that changes in number of RRVs does not affect the achievable rate of the channel with univesicular release since in this model, only one vesicle can be released from each terminal and the synaptic terminal has more than 1 RRV as it is shown in Fig. 5. Hence, the closed form equation derived in (17) gives the achievable rate of neuro-spike communication with multiple synaptic terminals, in which univesicular release model is used, even when the statistics of ready pool are not ignored. However, considering the changes in the number of RRVs decreases the achievable rate of the communication under multivesicular release 


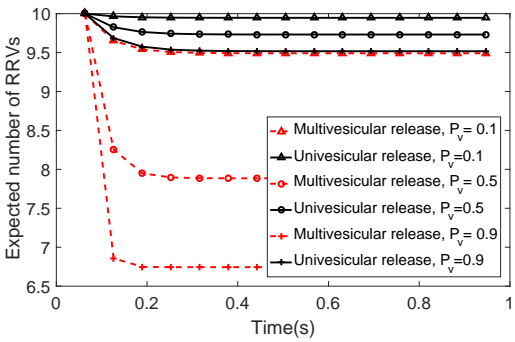

(a) $\lambda=15.85 \mathrm{~Hz}$

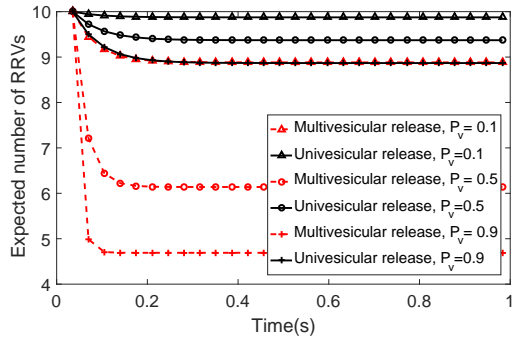

(b) $\lambda=28.49 \mathrm{~Hz}$

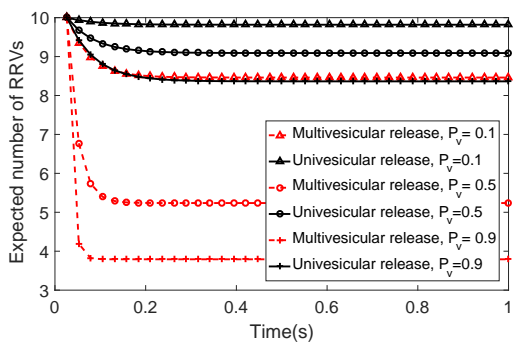

(c) $\lambda=38.04 \mathrm{~Hz}$

Fig. 5. The expected number of RRVs in a synaptic terminal under different vesicle release scenarios.

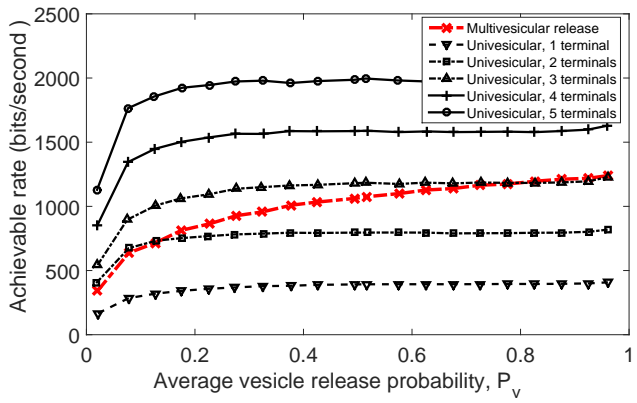

Fig. 6. Achievable rate as a function of average vesicle release probability when the number of RRVs vary due to the vesicle pool statistics.

model since all of the RRVs can release independently in this case and as it is shown in Fig. 5, the neuron is not able to recover all of the released vesicles under this model, hence it has less number of RRVs to release compared to the case that the neuron refills RRVs immediately.

The improvement in the achievable rate of neuro-spike communication by increasing number of synaptic terminals is depicted in Fig. 6, which gives us insight in designing a communication paradigm for nanomachines, for instance, it is informative in selecting number of connections that an artificial neuron needs to reliably transmit information in an artificial neural system or artificial synapse to a neuron.

\section{CONCLUSION}

In this paper, we derive the rate region of neuro-spike communication by considering the existence of multiple terminals with univesicular release model between an input and output neuron and using a more realistic pool-based model for vesicle release process. Moreover, we study the effects of the average vesicle release probability and the number of RRVs on the achievable rate and show that although all RRVs are free to release in the multivesicular release model, due to their limited number, the achievable rate of this model is less than the univesicular release model with multiple terminals. Furthermore, we see that the existence of multiple terminals between two neurons improves the synaptic connectivity between these neurons.

\section{REFERENCES}

[1] I. F. Akyildiz, et al., "Nanonetworks: A new communication paradigm," Computer Networks, vol. 52, no. 12, pp. 2260-2279, 2008.
[2] E. Balevi and O. B. Akan, "A physical channel model for nanoscale neuro-spike communications," IEEE Trans. Commun., vol. 61, no. 3 , pp. 1178-1187, 2013.

[3] O. B. Akan, et al., "Fundamentals of molecular information and communication science," Proceedings of the IEEE, 2016.

[4] M. F. Bear, et al., Neuroscience: Exploring the Brain, 3rd ed., 2007.

[5] M. Veletic, et al., "Peer-to-peer communication in neuronal nanonetwork," IEEE Trans. Commun., 2016.

[6] A. Manwani and C. Koch, "Detecting and estimating signals over noisy and unreliable synapses: information-theoretic analysis," Neural computation, vol. 13, no. 1, pp. 1-33, 2001.

[7] B. Maham, "A communication theoretic analysis of synaptic channels under axonal noise," IEEE Commun. Lett., vol. 19, no. 11, pp. 19011904, 2015.

[8] D. Malak and O. B. Akan, "A communication theoretical analysis of synaptic multiple-access channel in hippocampal-cortical neurons," IEEE Trans. Commun., vol. 61, no. 6, pp. 2457-2467, 2013.

[9] F. Gabbiani and C. Koch, "Coding of time-varying signals in spike trains of integrate-and-fire neurons with random threshold," Neural Computation, vol. 8, no. 1, pp. 44-66, 1996.

[10] D. Malak, et al., "Communication theoretic analysis of the synaptic channel for cortical neurons," Nano Communication Networks, vol. 4 no. 3, pp. 131-141, 2013.

[11] G. M. Shepherd and K. M. Harris, "Three-dimensional structure and composition of CA 3 CA1 axons in rat hippocampal slices: implications for presynaptic connectivity and compartmentalization," The Journal of neuroscience, vol. 18, no. 20, pp. 8300-8310, 1998.

[12] P. Andersen, "Chapter synaptic integration in hippocampal CA1 pyramids," Progress in brain research, vol. 83, pp. 215-222, 1990.

[13] E. P. Simoncelli, et al., "Characterization of neural responses with stochastic stimuli," The cognitive neurosciences, vol. 3, pp. 327-338, 2004.

[14] J. R. Geiger and P. Jonas, "Dynamic control of presynaptic ca 2+ inflow by fast-inactivating $\mathrm{k}+$ channels in hippocampal mossy fiber boutons," Neuron, vol. 28, no. 3, pp. 927-939, 2000.

[15] H. Ramezani and O. B. Akan, "A communication theoretical modeling of axonal propagation in hippocampal pyramidal neurons," IEEE Trans. Nanobiosci., 2016.

[16] V. Matveev and X.-J. Wang, "Implications of all-or-none synaptic transmission and short-term depression beyond vesicle depletion: a computational study," The Journal of Neuroscience, vol. 20, no. 4, pp. $1575-1588,2000$.

[17] J. De La Rocha and N. Parga, "Short-term synaptic depression causes a non-monotonic response to correlated stimuli," The Journal of Neuroscience, vol. 25, no. 37, pp. 8416-8431, 2005.

[18] A. A. Alabi and R. W. Tsien, "Synaptic vesicle pools and dynamics," Cold Spring Harbor perspectives in biology, vol. 4, no. 8, p. a013680, 2012.

[19] H. Ramezani and O. B. Akan, "Synaptic channel model including effects of spike width variation," in Proc. ACM NANOCOM, 2015, p. 11.

[20] A. J. Watt, et al., "Activity coregulates quantal ampa and nmda currents at neocortical synapses," Neuron, vol. 26, no. 3, pp. 659-670, 2000.

[21] T. Schikorski and C. F. Stevens, "Quantitative ultrastructural analysis of hippocampal excitatory synapses," The Journal of neuroscience, vol. 17 , no. 15 , pp. 5858-5867, 1997.

[22] E. A. Nimchinsky, et al., "The number of glutamate receptors opened by synaptic stimulation in single hippocampal spines," The Journal of neuroscience, vol. 24, no. 8, pp. 2054-2064, 2004. 